\title{
Inervación del Músculo Braquiorradial en Individuos Brasileños:Aspectos Anatomo-clínicos de la Biometría de Origen y Distribución Nerviosa
}

\author{
Innervation of the Brachioradialis Muscle in Brazilian Individuals: \\ Anatomo-clinical aspects of the Biometry of Origin and Nervous Distribution
}

Pérez Mérida, L. ${ }^{1,2}$; Melo, R. ${ }^{3}$; Sousa Rodriguez, C. F. ${ }^{4}$ \& Olave, E. ${ }^{5}$

PÉREZ MÉRIDA, L.; MELO, R.; SOUSA RODRIGUEZ, C. F. \& OLAVE, E. Inervación del músculo braquiorradial en individuos brasileños: Aspectos anatomo-clínicos de la biometría de origen y distribución nerviosa. Int. J. Morphol., 38(2):513-517, 2020.

RESUMEN: El nervio para el músculo braquiorradial (BR) ha sido utilizado en transferencias nerviosas para recuperaciones en funciones de la mano como consecuencias de lesiones que afectan el plexo braquial. Con el propósito de investigar el número y localización biométrica de los ramos primarios provenientes del nervio radial y puntos motores respecto un punto de referencia ubicado en la región del codo, se estudiaron 30 miembros superiores de individuos adultos brasileños, de la Universidad Estadual de Ciencias da Saúde de Alagoas, Maceió, Brasil. Las muestras se encontraban fijadas en solución de formaldehído al $10 \%$, de los cuales 15 miembros eran derechos y 15 izquierdos. Como punto de referencia se utilizó una línea que pasó a través de las partes más prominentes de los epicóndilos humerales, línea bi-epicondilea (LBE). Con respecto al origen nervioso para el músculo BR, todos los ramos se originaron a partir del nervio radial (NR). El promedio de la cantidad de Ramos Primarios (RP) fue de 1,53 (DS 0,73). En 18 muestras (60 \%) se observó sólo un RP; en 8 casos $(26,7 \%)$ se encontraron 2 RP, mientras que en 4 casos $(13,3 \%)$ de la muestra se observaron 3 RP. Sobre la localización biométrica de los orígenes de los RP, es importante mencionar que todos se ubicaron proximal a la LBE. En promedio, estos se ubicaron a $38 \mathrm{~mm}$ (DS 0,9); $29 \mathrm{~mm}$ (DS 1,2) y $22 \mathrm{~mm}$ (DS 1,0) para el primer, segundo y tercer RP, respectivamente. En relación a los puntos motores (PM), en 4 casos (13\%) se observó 1 PM dado por la penetración directa de un ramo primario, en 13 casos (43\%) existieron 2 PM, en 8 casos (27 \%) se encontraron 3 PM y en 5 casos (17\%) se observaron 4 PM. En tres casos (10 \%) la inervación hacia el músculo BR emitió filetes nerviosos hacia el músculo extensor radial largo del carpo. Los resultados expuestos en esta investigación son un importante aporte para bloqueos nerviosos, estimulaciones eléctricas y transferencias nerviosas.

PALABRAS CLAVE: Anatomía; Inervación; Puntos motores; Músculo braquiorradial; Biometría.

\section{INTRODUCCIÓN}

Las lesiones de plexo braquial distal, constituyen una de las causas de déficit funcional del miembro superior , con pérdida de la capacidad de prensión y pinza. En la última década ha surgido la posibilidad de realizar neurotizaciones, es decir, utilizar un nervio con representación cortical amplia, para reinervar otro territorio, usando una comunicación nerviosa termino-terminal o termino lateral a otro nervio, cuya función se requiere restaurar, ya sea de forma sensitiva o motora. En este contexto, el nervio para el músculo braquiorradial (BR) ha sido utilizado en transferencias nerviosas para recu- peraciones en funciones de la mano como consecuencias de lesiones que afectan el plexo braquial distal (C8 - T1) a través de su unión al nervio interóseo anterior (GarcíaLópez et al., 2011).

El músculo braquiorradial se origina en los $2 / 3$ proximales de la cresta supracondílea lateral y de la parte anterior del septo intermuscular lateral. Raramente este músculo es doble o ausente, pudiendo su tendón de inserción estar dividido en 2 o 3 fascículos (Tountas \& Bergman, 1993; Williams et al., 1995).

\footnotetext{
${ }^{1}$ Departamento de Anatomía y Medicina Legal, Facultad de Medicina, Universidad de Chile.

${ }^{2}$ Instituto de Investigación e Innovación en Salud, Facultad de Ciencias de la Salud, Universidad Central de Chile.

${ }^{3}$ Departamento de Ciencias Neurológicas Oriente, Instituto de Neurocirugía Asenjo, Universidad de Chile, Facultad de Medicina, Universidad de Chile.

${ }^{4}$ Facultad de Medicina de la Universidad Estadual de Ciencias da Saúde de Alagoas, Maceió, Brasil.

${ }^{5}$ Facultad de Medicina, Universidad de La Frontera, Temuco, Chile.
} 
La inervación del músculo braquiorradial proviene del nervio radial, que emite un ramo que ingresa al mencionado músculo en su tercio proximal y por su cara profunda (Tountas \& Bergman). Según Hovelacque (1927) este ramo se origina 3 a $4 \mathrm{~cm}$ distal al punto en donde ingresa el nervio radial a nivel del surco bicipital lateral, 5 o $6 \mathrm{~cm}$ proximal al epicóndilo lateral; para luego abordar al músculo por su cara medial (Rouvière \& Delmas, 1999).

Por su parte, Hosapatna et al. (2019) encontraron un ramo proveniente del nervio radial en $70 \%$ de la serie de 27 miembros superiores y 3 ramos en sólo una muestra, cuyo origen fue proximal a la línea bi-epicondilar (LBE). Este nivel de origen de estos ramos para el músculo en cuestión fue también descrito por Cho et al. (2013).

El propósito de este estudio fue investigar el número y localización biométrica de los ramos primarios provenientes del nervio radial y puntos motores respecto un punto de referencia ubicado en la región del codo.

\section{MATERIAL Y MÉTODO}

Se utilizaron 30 miembros superiores de individuos adultos brasileños, de la Facultad de Medicina de la Universidad Estadual de Ciencias da Saúde de Alagoas, Maceió, Brasil. Las piezas cadavéricas se encontraban fijadas en solución de formaldehído al $10 \%$, de los cuales 15 miembros eran derechos y 15 izquierdos. La disección de la región cubital se realizó con los miembros superiores en posición anatómica, en extensión y con material quirúrgico adecuado.

Luego de ubicar el nervio radial a nivel del surco bicipital lateral (Fig. 1), se procedió a medir el origen y distancia de los ramos primarios y puntos motores respecto a la línea biepicondílea (LBE), conformada por las partes más prominentes de los márgenes, lateral y medial, de ambos epicóndilos humerales. A partir de esta línea se midió con huincha métrica, el origen del o los ramos que se dirigían al músculo, así como también las distancias entre ésta y los puntos motores que penetraban a nivel muscular.

\section{RESULTADOS}

Con respecto al origen nervioso para el músculo BR, todos los ramos se originaron a partir del NR.

El promedio de la cantidad de ramos primarios (RP) fue de 1,53 (DS 0,73). En 18 muestras (60\%) se observó sólo un RP (Fig. 2); en 8 casos (26,7 \%) se encontraron 2 RP (Fig. 3), mientras que en 4 casos $(13,3 \%)$ de la muestra se observaron 3 RP.

Sobre la localización biométrica de los orígenes de los RP, es importante mencionar que todos se ubicaron sobre la LBE (proximal). En promedio, estos se ubicaron a 38

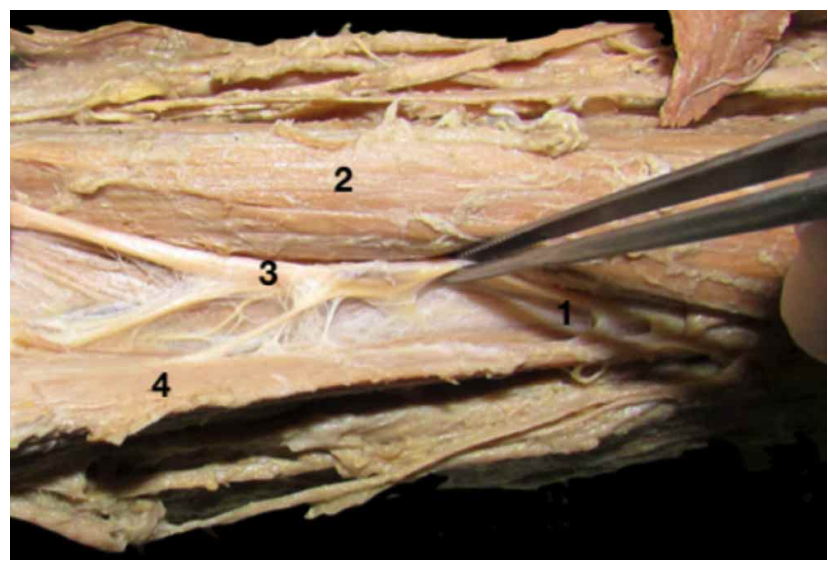

Fig. 1. Surco bicipital lateral: 1. Aparición del nervio radial a nivel del surco; 2. M. braquial; 3. N. radial; 4. M. braquiorrradial.

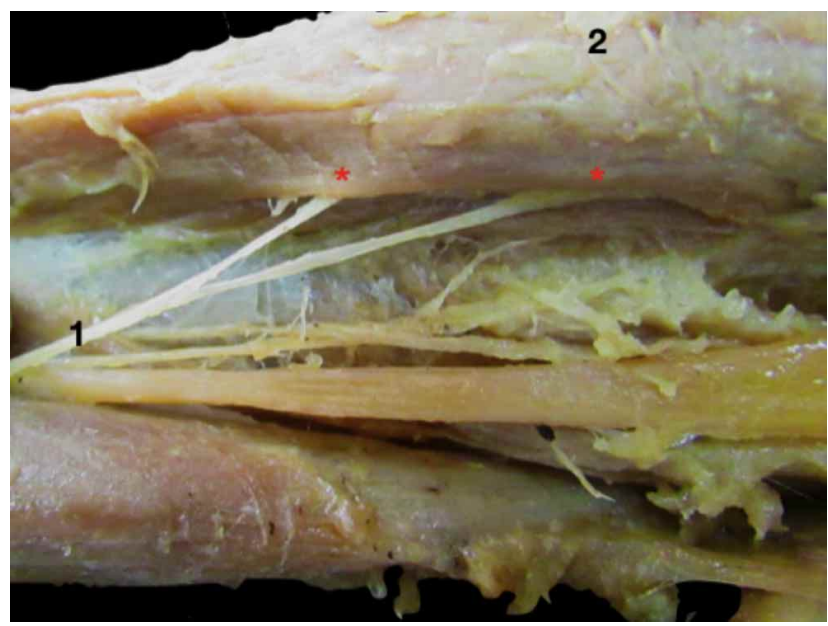

Fig. 2. Origen del nervio para el M. braquiorradial a partir de un RP. 1. Nervio para el M. braquiorradial; 2. M. braquiorradial. Estrellas de color rojo corresponden a PM.

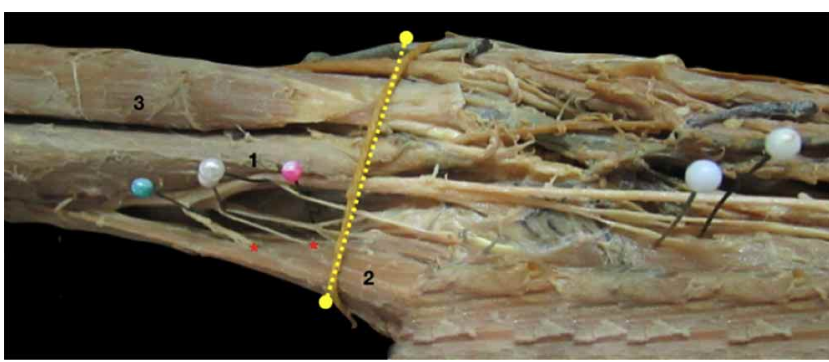

Fig. 3. Origen de los Nn. para el M. braquiorradial a partir de dos RP. 1. M. braquial; 2. M. braquiorradial; 3. M. bíceps braquial. Estrellas de color rojo corresponden a PM; línea amarilla corresponde a LBE. 
mm (DS 0,9); 29 mm (DS 1,2) y $22 \mathrm{~mm}$ (DS 1,0) para el primer, segundo y tercer RP, respectivamente. El origen más proximal se ubicó a $55 \mathrm{~mm}$, mientras que el más distal se ubicó a $10 \mathrm{~mm}$ sobre la LBE.

Los ramos secundarios (RS) se encontraron en promedio 2,5 (DS 0,94) y fueron éstos específicamente, los que penetraban a nivel muscular transformándose en puntos motores (PM).

En 4 casos (13\%) se observó 1 PM dado por la penetración directa de un ramo primario, en 13 casos (43\%) existieron 2 PM, en 8 casos (27 \%) se encontraron 3 PM y en 5 casos (17\%) se observaron 4 PM (Fig. 4).

Independiente la cantidad de PM, el primer PM se ubicó en promedio a $20 \mathrm{~mm}$ (DS 1,7) proximal a la LBE. El segundo PM penetró a nivel muscular a 12,5 mm (DS 1,22), mientras que el tercer PM se localizó en promedio a $10 \mathrm{~mm}$ (DS 1,63). Finalmente cuando se observó un cuarto PM, éste se ubicó a 15 mm (DS 1,41) distal al LBE. Cuando se visualizó un cuarto PM que corresponde al $17 \%$ de la muestra es importante recalcar que fue el único de los 4 PM que se ubicó distalmente a la LBE.

El rango de penetración del PM más proximal fue de $50 \mathrm{~mm}$ proximal a la LBE y el más distal se ubicó a $40 \mathrm{~mm}$ distal a la LBE.

En tres casos (10\%) la inervación hacia el BR emitió filetes nerviosos hacia el músculo extensor radial largo del carpo (ERLC) (Fig. 5). Finalmente, en un caso, el nervio hacia el músculo ERLC se originó mas proximal en comparación con el origen del nervio para el músculo BR.

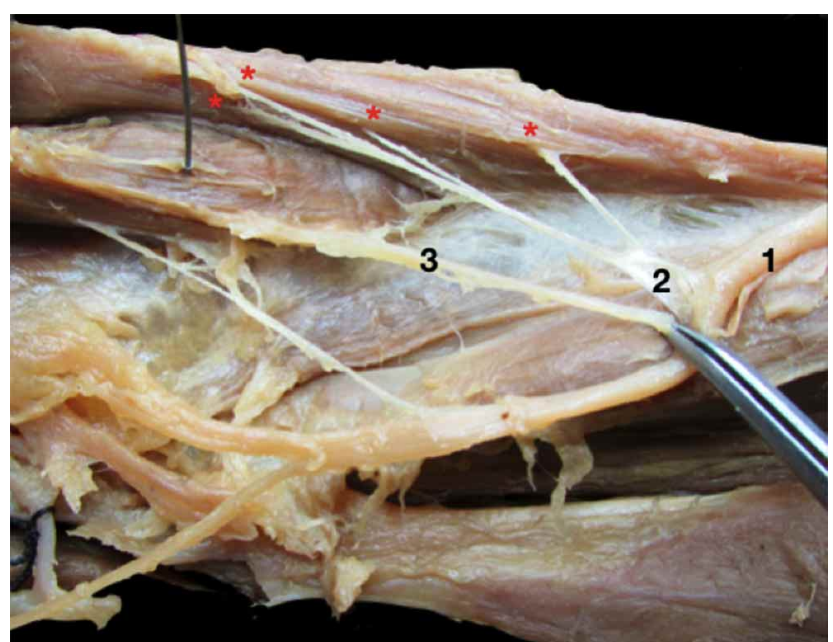

Fig. 4. Penetración de 4 PM a nivel muscular. 1. N. radial; 2. RP del M. braquiorradial; 3. N. para el M extensor radial largo del carpo. Estrellas de color rojo corresponden a PM.

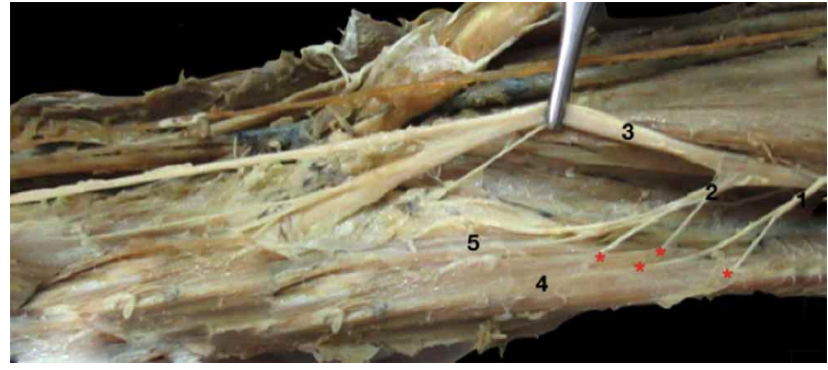

Fig. 5. 1. RP para el M. braquiorradial; 2. Tronco común para los Mm. braquiorradial y extensor radial largo del carpo; 3. N. radial; 4. M. braquiorradial; 5. M. Extensor radial largo del carpo. Estrellas de color rojo corresponden a PM.

\section{DISCUSIÓN}

En el presente estudio, la inervación del músculo BR provenía directamente del NR y recibió en promedio 1,5 RP. En el $60 \%$ de nuestra serie se identificó un RP único mientras que en $26,7 \%$ se observaron 2 RP, y el $13,3 \%$ restante se presentó con 3 RP. En promedio estos RP se ubicaron a 38, 29 y $22 \mathrm{~mm}$ próximal al LBE, mientras que el origen del ramo más proximal se ubicó a $55 \mathrm{~mm}$ y el más distal a $10 \mathrm{~mm}$, ambos proximales a la LBE. Latev \& Dalley (2005) que estudiaron 43 muestras, encontraron en 20 casos $(46,5 \%)$ un RP. En promedio el punto de origen se ubicó a 30 mm (DS 10,6) próximal al epicóndilo lateral (EL). En 17 casos (40\%) hubo 2 RP. El origen próximal del primer ramo primario estuvo a $30 \mathrm{~mm}$ (DS $16,8 \mathrm{~mm}$ ) mientras que el origen del segundo ramo primario se ubicó a $15 \mathrm{~mm}$ (DS 18,9). De éstos, en 7 muestras uno o ambos RP se dividieron en ramos secundarios. En los restantes 6 casos (14\%) hubo 3 RP procedentes del nervio radial. En promedio los puntos de origen se localizaron a 44, 34 y $26 \mathrm{~mm}$ proximales al EL, respectivamente.

En estudio realizado por Hosapatna et al. (27 miembros superiores), el origen de los RP para el músculo BR estuvo entre $63 \mathrm{~mm}$ y $22 \mathrm{~mm}$ proximal a LBE así como también Cho et al. (40 muestras) indicaron que el origen de los RP fue siempre proximal a la LBE.

El promedio de PM para Latev \& Dalley fue de 2,7 ( 1 a 4), valor con el que concordamos, ya que registramos 2,5 PM en nuestra investigación y se acerca a los encontrados por Abrams et al. (1997) cuyo promedio de PM o ramos secundarios fue de 2,9 (DS 1,1).

Con respecto a la distancia de los PM respecto al EL, los estudios de Latev \& Dalley, registraron lo siguiente: cuando hubo 1 ramo ingresando en el músculo (4 casos) lo hizo 
en un promedio de $1,0 \pm 8,5 \mathrm{~mm}$, proximal al EL; cuando hubo 2 ramos entrando en el músculo (19 casos) lo hicieron en promedio de $10 \pm 18,6 \mathrm{~mm}$, proximal al EL, el ramo más proximal y $8 \pm 24,7 \mathrm{~mm}$, distal al LE, el ramo más distal,; Cuando se presentaron 3 ramos ingresando en el músculo ( 8 casos) el promedio de entrada de esos ramos fueron, de proximal a distal, $27 \pm 13,9,13 \pm 10,4 \mathrm{~mm}$, proximal al LE, mientras que el 3er ramo fue $5 \pm 7,6 \mathrm{~mm}$ distal al LE. Ya nuestro estudió arrojó en promedio, valores de $20 \mathrm{~mm}$ para el primer PM, 12,5 mm para el segundo, y, $10 \mathrm{~mm}$ para el tercer PM, proximales a la LBE; sólo cuando hubo un cuarto PM (16,7\% de la muestra), éste se ubicó $15 \mathrm{~mm}$, distal a la LBE.

Por otro lado, nuestros resultados se aproximan a los vistos por Liu et al. (1997), que en su muestra de 10 miembros superiores, el promedio de RP fue de 1,2 (DS 0,42), mientras que el promedio de PM fue 2,4 (DS 0,70). El promedio de 1,5 RP de nuestro estudio concuerda con Zhang et al. (2016), que en 28 muestras obtuvieron un promedio de 1,5. El primer RP se ubicó a 3,13 cm (DS 1,12) proximal al EL.

Al comparar nuestro estudio con los de Bertone et al. (2008) que realizó una serie de 30 casos, concordamos con los porcentajes informados para 2 y $3 \mathrm{RP}$, respectivamente, pero su resultado para $1 \mathrm{RP}$ es mucho menor (11 casos, $36,6 \%$ ) que el $60 \%$ de nuestra serie. Adicionalmente, encontraron que en 8 casos $(26,7 \%)$ había un tronco común para el músculo braquiorradial y el músculo extensor radial largo del carpo.

Sobre el rango de origen nervioso de los RP, nuestra investigación se asemeja a lo informado por Sunderland (1946), quien encontró el punto de origen nervioso más alto o proximal, $60 \mathrm{~mm}$ proximal al epicóndilo y el más bajo o distal a $15 \mathrm{~mm}$ respecto del EL, mientras que en el presente estudio se ubicaron a 55 y $10 \mathrm{~mm}$, respectivamente.

Kerver et al. (2013), mencionaron en su muestra de 20 miembros, 1 a 2 RP originados directamente del NR y un promedio de 2 PM (rango de 1 a 3 ). Finalmente, Matavelli Júnior et al. (2019) identificaron en 10 casos (70 \%) 7 ramos primarios únicos y en 3 muestras (30\%) 2 RP. En 2 casos $(20 \%)$ se encontró un tronco común con filetes nervios hacia el ERLC, que se relacionan con un $10 \%$ de nuestra muestra que originó un tronco común para inervación de ambos músculos.

Finalmente y al igual que Branovacki et al. (1998) pudimos observar un origen proximal del nervio para el ERLC sobre el origen del nervio para BR, que en su muestra alcanzó un $10 \%$ y que en nuestro estudio alcanzó 3,3\% correspondiente sólo a 1 caso.
En tres casos (10 \%) del presente estudio la inervación hacia el BR emitió filetes nerviosos hacia el músculo ERLC, (Fig. 5), algo semejante y ya visto anteriormente (Pérez Mérida et al., 2019) en donde la inervación para el músculo ERLC algunas veces era compartida con la del músculo BR.

Como hemos visto en este trabajo y a la luz de las investigaciones, tanto la cantidad de ramos primarios como de puntos motores para el músculo BR es variable. Sin duda, un alto porcentaje presenta al menos 1 o 2 ramos primarios, mientras que un buen porcentaje muestra también 2 PM, encontrándose hasta 4 . El rango de penetración de estos ramos nerviosos abarca una distancia de $90 \mathrm{~mm}(50 \mathrm{~mm}$ proximal y $40 \mathrm{~mm}$ distal a LBE), que son claros de visualizar para cualquier intervención que se realice a nivel del margen medial de este músculo y también a nivel del codo. Por otro lado, es importante recalcar que los nervios que se dirigen al músculo braquiorradial avanzan algunos milímetros por la superficie muscular, penetrando luego al músculo.

Los resultados expuestos en esta investigación son un importante aporte para bloqueos nerviosos, estimulaciones eléctricas y transferencias nerviosas que se puedan realizar en el País.

Sin duda, aún faltan estudios biométricos de éste y otros nervios de la región del codo, que seguramente aportarán un detallado conocimiento morfológico a las intervenciones neuroquirúrgicas en el miembro superior.

PÉREZ MÉRIDA, L.; MELO, R.; SOUSA RODRIGUEZ, C. F. \& OLAVE, E. Innervation of the brachioradialis muscle in Brazilian individuals: Anatomo-clinical aspects of the biometry of origin and nervous distribution. Int. J. Morphol., 38(2):513-517, 2020

SUMMARY: The nerve for the brachioradialis muscle (BR) has been used in nerve transfers for recoveries in functions of the hand as a consequence of lesions affecting the brachial plexus. With the purpose of investigating the number and biometric location of the primary branches coming from the radial nerve and motor points with respect to a reference point located in the elbow region, thirty upper limbs of Brazilian adult individuals from the State University of Sciences of Saúde de Alagoas, Maceió, Brazil were used. The samples were fixed in $10 \%$ formaldehyde solution, of which 15 were right and 15 left. As a reference point, a line was used that passed through the most prominent parts of the humeral epicondyls, bi-epicondilar line (BEL). With respect to the nervous origin for the BR muscle, all the branches originated from the radial nerve $(\mathrm{RN})$. The average number of primary branches $(\mathrm{PB})$ was 1.53 (SD 0.73). In 18 samples $(60 \%)$ only one PB was observed; in 8 cases $(26.7 \%) 2 \mathrm{~PB}$ were found, while in 4 cases $(13.3 \%)$ of the sample 3 PB were observed. Regarding the biometric location of the origins of $\mathrm{PB}$, it is important to mention that all 
were located proximal to the BEL. On average, these were located at $38 \mathrm{~mm}$ (SD 0.9); $29 \mathrm{~mm}$ (DS 1.2) and $22 \mathrm{~mm}$ (DS 1.0) for the first, second and third $\mathrm{PB}$, respectively. In relation to the motor points (MP), in 4 cases $(13 \%) 1 \mathrm{MP}$ was observed as direct penetration of the $\mathrm{PB}$, in 13 cases $(43 \%)$ there were $2 \mathrm{MP}$, in 8 cases $(27 \%)$ they found $3 \mathrm{MP}$ and in 5 cases (17\%) $4 \mathrm{MP}$ were observed. In three cases $(10 \%)$ the innervation towards the BR muscle emitted nerve fillets towards the extensor carpi radialis longus muscle. The results presented in this investigation are an important contribution to nerve blocks, electrical stimulations and nerve transfers.

KEY WORDS: Anatomy; Innervation; Motor points; Brachioradialis muscle; Biometry.

\section{REFERENCIAS BIBLIOGRÁFICAS}

Abrams, R. A.; Ziets, R. J.; Lieber, R. L. \& Botte, M. J. Anatomy of the radial nerve motor branches in the forearm. J. Hand Surg. Am., 22(2):232-7, 1997.

Bertone, V. H.; Ottone, N. E.; Lo Tártaro, M. A.; García de Quiros, N.; Arrotea, A.; Domínguez, M.; Finkelstein, D.; López Bonardi, P.; Shinzato, S. \& Medan, C. Anatomical and clinical considerations about the radial nerve in the elbow. Int. J. Morphol., 26(2):437-44, 2008.

Branovacki, G.; Hanson, M.; Cash, R. \& Gonzalez, M. The innervation pattern of the radial nerve at the elbow and in the forearm. $J$. Hand Surg. Br., 23(2):167-9, 1998.

Cho, H.; Lee, H. Y.; Gil, Y. C.; Choi, Y. R. \& Yang, H. J. Topographical anatomy of the radial nerve and its muscular branches related to surface landmarks. Clin. Anat., 26(7):862-9, 2013.

García-López, A.; Sebastian, P.; Martinez, F. \& Perea, D. Transfer of the nerve to the brachioradialis muscle to the anterior interosseous nerve for treatment for lower brachial plexus lesions: case report. J. Hand Surg. Am., 36(3):394-7, 2011.

Hosapatna, M.; Souza, A. D.; Rao, M. \& Hari Ankolekar, V. Morphology and innervation of brachioradialis and flexor carpi radialis and their utility in tendon transfer surgeries: a cadaveric study. Morphologie, 2019. doi: 10.1016/j.morpho.2019.10.046. Online ahead of print.

Hovelacque, A. Anatomie des Nerfs Crâniens et Rachidiens et du Système Grand Sympathique Chez l'Homme. Paris, G. Doin et cie, 1927. pp.498.

Kerver, A. L. A.; Carati, L.; Eilers, P. H. C.; Langezaal, A. C.; Kleinrensink, G. J. \& Walbeehm, E. T. An anatomical study of the ECRL and ECRB: feasibility of developing a preoperative test for evaluating the strength of the individual wrist extensors. J. Plast. Reconstr. Aesthet. Surg., 66(4):543-50, 2013.

Latev, M. D. \& Dalley 2nd, A. F. Nerve supply of the brachioradialis muscle: surgically relevant variations of the extramuscular branches of the radial nerve. Clin. Anat., 18(7):488-92, 2005.

Liu, J.; Pho, R. W.; Pereira, B. P.; Lau, H. K. \& Kumar, V. P. Distribution of primary motor nerve branches and terminal nerve entry points to the forearm muscles. Anat. Rec., 248(3):456-63, 1997.

Matavelli Júnior, F. C.; Gobbi, L.; dos Santos, M. P. S.; Caetano, E. B.; Vieira, L. A. \& de Andrade, R. A. Transfer of the radial nerve branches for the treatment of the anterior interosseous nerve lesion: an anatomical study. Acta Ortop. Bras., 27(6):298-303, 2019.

Pérez-Mérida, L.; Sousa-Rodrigues, C. F. \& Olave, E. Innervation of the extensor carpi radialis longus muscle in brazilian individuals: biometry of its origin and distribution. Int. J. Morphol., 37(2):423-7, 2019.

Rouvière, H. \& Delmas, A. Anatomía Humana: Descriptiva, Topográfica y Funcional. 10a ed. Paris, Masson, 1996.

Sunderland, S. Metrical and non-metrical features of the muscular branches of the radial nerve. J. Comp. Neurol., 85:93-111, 1946.

Tountas, C. \& Bergman, R. Anatomic variations of the upper extremity. New York, Churchill \& Livingstone, 1993.

Williams, P.; Warwick, R.; Dyson, M. \& Bannister, L. Gray Anatomía. 37a ed. Rio de Janeiro, Guanabara Koogan, 1995.

Zhang, L.; Dong, Z.; Zhang, C. L. \& Gu, Y. D. Surgical anatomy of the radial nerve at the elbow and in the forearm: anatomical basis for intraplexus nerve transfer to reconstruct thumb and finger extension in C7 - T1 brachial plexus palsy. J. Reconstr. Microsurg., 32(9):670-4, 2016.

\author{
Dirección para Correspondencia: \\ Luis Pérez-Mérida, Mg.Cs.,Morfología \\ Departamento de Anatomía y Medicina Legal \\ Facultad de Medicina \\ Universidad de Chile \\ Santiago \\ CHILE
}

\section{Email: luispemerida@gmail.com enrique.olave@ufrontera.cl}

Recibido : 10-10-2019

Aceptado: 01-12-2019 